\title{
Fractures in old age: a public health perspective
}

Witold Pawłowski ${ }^{1}$, Krzysztof Goniewicz ${ }^{2}$, Mariusz Goniewicz ${ }^{3}$, Dorota Lasota ${ }^{4}$

'Department of Disaster Medicine, Medical University of Warsaw, Warsaw, Poland ${ }^{2}$ Department of Security Studies, Polish Air Force Academy, Poland

${ }^{3}$ Department of Emergency Medicine, Medical University of Lublin, Lublin, Poland ${ }^{4}$ Department of Experimental and Clinical Pharmacology, Medical University of Warsaw, Warsaw, Poland

Submitted: 27 July 2018

Accepted: 12 September 2018

Arch Med Sci Aging 2018; 1: e19-e24

DOI: https://doi.org/10.5114/amsa.2018.79431

Copyright $\Subset 2018$ Termedia \& Banach

\section{Abstract}

Fractures of old age are fractures occurring in persons with low resistance of osseous tissue due to diseases such as osteoporosis. The most common osteoporotic fractures are fractures of the spine, proximal femur, distal radius as well as some diaphyseal fractures seen by orthopaedic surgeons. The research on the basis of domestic and foreign literature and the results of their observations are presented as well as the principles of epidemiology for the identification and treatment of age-related fractures. Almost half of falls in old age lead to injuries which are often the cause of hospitalization. Falls are a major cause of disability and the fifth most frequent cause of death in people over 75 years. Treatment of age-related fractures should ensure the possibility of prompt return of the patient to independent movement and performing daily activities.

Key words: aging, fractures, public health.

\section{Introduction}

Fractures are one of most common post-traumatic injuries of the osteoarticular system. They occur when an injury force is larger than the mechanical resistance of bones. Size, shape and consistency of bones change in the course of time. Fractures of bones, which in the case of the elderly are stiff and often changed by osteoporosis, occur as the result of weaker strength than in the case of younger people. Older people more often suffer from comminuted fractures [1-4].

A fracture is a breakage of bone tissue integrity through its whole section. Fracture can occur in one or several bones, even distant from each other. In respect of the character of a fracture, they can be divided into the following types: Open fractures - breakage of osseous tissue integrity together with injury of integument; there is contact of the bone with the outside environment. Closed fractures - breakage of osseous tissue integrity without integument injury - there is no contact between fractured bone and the external environment. In respect of fracture mechanism, they divide into: fractures resulting from a direct injury, when the injury force impacts directly on a bone; fractures resulting from an indirect injury when injury force has an indirect impact on a bone through e.g. other bone, joint, sinew [1-4].

In the case of injuries indirectly acting on a bone, a fracture can result from mechanisms such as pulling, bending, spraining or squeezing. Frac-
Corresponding author:

Witold Pawłowski

Department of

Disaster Medicine

Medical University

of Warsaw

81 Zwirki i Wigury St

02-091 Warsaw, Poland

E-mail: witold.pawlowski@ wum.edu.pl 
tures resulting from pulling most often result from a violent muscle or ligament tear and breaking its osseous attachment. The fracture space is in most cases perpendicular to the direction of the breaking force. Fractures resulting from bending arise due to a lever mechanism. The force acts perpendicularly to a bone supported at both its ends or on one of them while the other is stabilized. Such a fracture may also occur when two forces act on a stabilized bone. In the case of the bending mechanism, oblique fractures are the most common. Spiral fracture is characterized when one of the bone's ends is stabilized and torsional force acts on the other end. The fracture gap in this type is spiral. Fractures resulting from crushing occur in trabecular bones because of flexure or compression mechanisms. Oblique fractures, transverse fractures or fractures with an indirect wedge shape break off. Serious injuries may result in disaggregation or crushing of bones [1-4]. In respect of fraction gap alignment, the following types of fractures can be distinguished: oblique, spiral, transverse, comminuted, etc. The fracture gap can go through the whole bone intersection or only its part. There are fractures with or without splinter dislocations. Fractures without dislocations mostly occur in young people as their thick and flexible periosteum prevents splinters from dislocating [1-4].

In respect of location, there are fractures of shaft, metaphysis, epiphysis, osseous protrusions and joint surfaces. Fractures can be complicated or not complicated. Complications can occur at the time of an injury or during treatment. The most common are primary complications of vessels, nerves, brain, spinal cord and internal organs. Secondary complications include delayed adhesion, false joints, aseptic necrosis, adhesion in improper orientation, contractures, osteitis, compartment syndromes, paralysis, and bedsores. A specific form of fractures is pathologic fracture. It results from a minor injury and sometimes without a tangible injury in pathologically changed osseous tissue. Causes of such fractures include tumor metastases to bones, decalcification or atrophy of osseous tissue, osteoporosis and osseous tissue inflammations. In general, every bone can undergo a pathological fracture. However, the most dangerous are fractures of the neck of the femur and multiple compacted fractures of vertebrae [4-8].

\section{Spine injuries}

One of the most serious injuries that occur in the osteoarticular system are spine injuries. In most cases, such injuries are located in a cervical vertebra or at a joint between a thoracic vertebra and a lumbar vertebra $[9,10]$. Such injuries de- pend mostly on the injury strength and impacted place. They can be accompanied by shock and lesions of the spinal cord, brain, thoracic organs, and abdominal organs [11].

Spinal cord injuries can occur individually but in most cases they occur with injuries of other parts of the body, e.g. cervical vertebra injuries and head lesions. The most common causes of spinal cord injuries are falls from heights and accidents. Other causes include unfortunate dives, crushes, and impacts. One should suspect spinal cord injuries in the case of every unconscious or head wounded person [12]. In the case of spinal cord injuries, first aid should be performed in such way that secondary injuries, dislocations and vertebral compressions do not occur. The injured person should be transported in a supine position on a firm base such as a stiff stretcher. During transport, the patient should be well secured to a stretcher in order to limit movements and prevent further injuries. In the case of cervical vertebra injuries, it is important to protect this part so that side movements are impossible. It should be done using a collar, sand bags or using traction behind the head. A patient suspected of having spinal cord injury should be transported with the assistance of several persons, each being responsible for stabilizing a particular section [13].

Symptoms of fractures vary depending on location and bone function, strength of attached muscles, type of fracture, spread of lesions in surrounding tissues and time since the moment of injury.

In the case of post-traumatic injuries of locomotor organs, the main procedures at the accident place mainly involve life saving actions, health protection, limitation of injury effects and providing care for a patient. In such a case, the most important matters are evaluation of the general patient's state and determination of procedures' order, airway management, ensuring sufficient breathing, help in breathing, ensuring proper functioning of the patient's circulatory system, stemming the bleeding, dressing wounds and immobilizing the locomotive organ, implementation of anti-shock procedures, and calling a specialist service in order to transport a patient as fast as possible to the medical center where he or she can receive full medical service [1-4].

In order to properly take care of the injured organ, one should evaluate the situation, safety and general state of a patient, call the specialized medical service, calm down a patient, lay a patient in a supine position, arm reclining position or sitting position, precisely and carefully conduct a secondary test of a patient, undress a patient or dissect clothes in the injury area, dress wounds and massive external bleeding, temporally immobilize a patient before transporting, conduct im- 
mobilizing according to Pott's rules: rule I: in the case of long bone fractures, at least two adjacent joints should be immobilized; rule II: in the case of joint injuries, bones constituting that joint should be immobilized; immobilize the limb in a physiological position, immobilize a limb in a position after an injury in the case of an open fracture, when a limb is in pathologic position or when a closed fracture can transform into an open one as the result of a splinter stressing skin, raise a limb above the heart level, support an upper limb in a sling, keep fingers or toes uncovered, provide further care for a patient [14].

In hospital conditions, two basic methods of fracture treatment are used: operating and non-operating. The non-operating procedure mostly consists in anatomic bone setting, splinter immobilizing until their stable adhesion and fast rehabilitation in order to obtain full functionality of injured limb. A limb setting is done under the following anesthesia types: general, peridural, perineural or local. In order to set a broken limb, the following techniques are used: axial traction, splinter setting or dislocations raising. Setting requires proper tables, splints, traction equipment, direct and indirect tractions [14].

The second method of treatment is operational. Such treatment is used in the case of open fractures, fractures that cannot be set conservatively, and fractures with complications such as lesions of vessels, nerves and internal organs. Fractures that might be treated operationally include fractures that can be set conservatively but threatening displaced fragments of bone fractures [14].

Methods of internal and external osteosynthesis are also used in operational treatment. Osteosynthesis is the process of synthesis of bones. It enables treatment without external immobilization, which is helpful in early rehabilitation. Stabilization through external osteosynthesis enables immobilization and correction of osseous fragments. This method prevents metal entering into the fracture focus, which differentiates it from internal synthesis [15]. Bodily ageing is an inextricable process. Changes are irreversible. Functions of almost every organ are impaired. There are also many negative changes from the morphological point of view. For example, hormone management is disordered, bone mineral density is gradually decreasing, bone immunity is lower and fracture risk is higher $[16,17]$.

It is difficult to specify a unique definition of old age, also called senescence. Undoubtedly, it has to be considered in relation to the ageing process. Like senescence itself, also its beginning cannot be strictly determined. According to the WHO classification includes three sub-stages: $60-75$ years old is ripe old age, 76-90 is old age and beyond 90 is hoary age $[6,9,12]$. According to the classifi- cation of Anglo-Saxon gerontologists, old age encompasses the period between the $65^{\text {th }}$ and $95^{\text {th }}$ years of life $[18,19]$.

\section{Fractures of old age}

Fractures of old age are fractures occurring in persons with low resistance of osseous tissue due to diseases such as osteoporosis. Most often, they occur as a result of a low-energy injury. Such fractures can occur in persons in their sixties. "Fractures are a clinical symptom of osteoporosis and serious factor for further fractures in the future" [20]. Osteoporotic fractures are one of the most frequent causes of disabilities and are very costly part of health care systems' budgets in many regions of the world. Epidemiological data show that in Poland, more than $25 \%$ of the population of people over 50 years old are threatened with osteoporotic fractures, and fractures of a root close to a thigh bone constitute $9 \%$ of reasons of hospitalizations at orthopedic wards. In the USA, 25 million people are afflicted by osteoporosis. It is the cause of 1.3 million fractures a year, including 500 thousand spinal cord fractures, 250 thousand thigh bone fractures and 240 thousand antebrachial bone fractures. According to the WHO report, the number of thigh bone fractures in the world will amount to 4 million in 2025 and over 6 million in 2050. As present data show, if proper preventive actions are not taken now, osteoporotic fractures and their causes will be more frequent [21, 22].

The risk of osteoporotic fractures is higher in the case of women $-46.4 \%$ for 50 -year old women and $22.4 \%$ for 50 -year old men. After fractures of the proximal end of the thigh bone of elderly people, $20 \%$ of women and $30 \%$ of men die due to complications and $50 \%$ of those who survive becomes disabled. $25 \%$ of women beyond their fifties suffer from spinal cord fractures, $60 \%$ of which are asymptomatic. Fractures of the proximal end of the thigh bone occur in $0.25 \%$ of women. According to the National Health Service Fund in Poland data, 15888 persons in their fifties in Poland were given treatment due to proximal end of the thigh bone fractures [23].

Falls are one of the main causes of disabilities and they constitute $25 \%$ of all causes of deaths in persons over 75 years of age. About $40 \%$ of persons over 60 years old and $50 \%$ of healthy people over 80 years old suffer from a fall at least once a year. Falls are a direct cause of $100 \%$ of radial bone fractures, $90 \%$ of neck of femur fractures and $25 \%$ of spinal cord fractures [24].

Vertebral body fractures are also called compacted spinal cord fractures. There are two types of spinal cord fractures: stable and instable. Stable fractures are characterized by trabecular bone compression and wedge-shaped deformation of 
the vertebral body together with limiting plates impairment. Parts that are not impaired are intervertebral discs, back vertebra wall and spinal cord ligament. Instable fractures occur as a result of a bending-rotary mechanism. Instable fractures are characterized by a subluxation within the spinal cord's locomotor segment with vertebral canal contraction, breakage of a back ligament apparatus and impairment of the back wall of the vertebral centrum and intervertebral disc. Vertebrae that most often are fractured are the twelve thoracic vertebrae and $1^{\text {st }}, 2^{\text {nd }}$ and $3^{\text {rd }}$ lumbar vertebrae. It is related to higher mobility of lumbar vertebrae as thoracic vertebras are stabilized by the thoracic cavity [8]. Vertebral body fractures, which are related to osteoporotic changes in bones, result from minor injuries that may happen during everyday activities: getting up, sinking into a chair, tripping or sneezing. Only $25 \%$ of fractures result from falls [24]. The most frequent localizations of osteoporotic fractures are vertebrae Th8-Th12. They include wedge fractures and biconcave fractures.

Osteoporotic fractures of vertebra bodies are stable fractures but are also related to risk of another fracture. They are fractures showing no clinical symptoms in $60 \%$ of patients. Clinical symptoms include sharp pain, easy to locate, local pressure pain and sometimes radicular syndrome. Vertebral body fractures manifest themselves with increase of thoracic kyphosis, i.e. back rounding, the so-called "widow's hump" [24].

Diagnosis of osteoporotic fractures should include, apart from anamnesis, conventional X-ray examination [10]. Fracture treatment is most often conservative and sometimes operational. Conservative treatment involves applying a plaster jacket in order to limit spinal cord movements and decrease pain [2, 8]. Long-term jacket wearing is harmful and causes the risk of amyotrophy and further osteoporosis. It is not recommended to apply plaster jackets in the case of osteoporotic fractures [25]. Jackets should not be used for multiple bodies fractures, for bedridden patients and patients with respiratory disorders. During the period just after an injury, often intensified pain is observed and it is suggested to hospitalize a patient for a period of 3 to 5 days, and use analgesic treatment with non-steroid anti-inflammatory medicines and opioids. Operational treatment consists in filling the interior of a vertebral body with bone cement via a needle. Sometimes, high treatment costs limit availability of this method [25]. After a fracture, the rehabilitation period consists in muscle training in order to prevent spinal cord deformations, movement coordination practice, physiotherapy and acupuncture. Treatment prognoses and results are at a good level [25].
Fractures of distal radial bone or so-called fracture in a typical place occur as a result of a fall from body height and attempt of arm support [25]. There are two types of such fractures: Colles' fracture, when a person falls on an outstretched arm, and Smith's fracture, when a person falls on a flexed wrist [8]. Colles' fractures constitute up to $90 \%$ of all wrist fractures when the arm was in the back flexed position. Fracture of the distal radius bone is diagnosed on the basis of pain at wrist section, painful movement limits and wrist's sensitiveness and edema. Fracture is diagnosed on the basis of an X-ray picture.

The most frequent treatment of wrist fractures is based on conservative treatment, i.e. local anesthesia, manual reposition of splinters and dressing a plaster. Instable fractures are to be treated operationally. Nowadays, operational treatment is used in the case of wrist fractures. The aim of this treatment is faster rehabilitation of an impaired limb after adhesion and shorter or even lack of plaster use. Prognoses and results of such treatment are generally at a good level. Complications include growth disorders and carpal tunnel syndrome, i.e. median nerve compression, sinew impairments and inflammation of vagina tendinis musculi flexoris pollicis longi [8]. About $30 \%$ of fractures are complicated with Sudeck's atrophy. Anti-complication prevention is based on cutting plaster, raising a limb and isometric practice of forearm muscles. After removing the plaster, the range of practice should be extended with wrist and elbow joint active movements and active resistive exercises [19]. Fractures of the volar end of the thigh bone can be divided into fractures of: thigh bone head, thigh bone neck and trochanteric. Thigh bone neck fractures are very frequent in persons beyond their sixties and account for $30 \%$ of all fractures. They are the most serious result of osteoporosis. They can result in very serious complications such as pneumonia, cystitis, circulatory-respiratory failures, congestions in the brain, protein management disorders and bedsores. It is commonly called "a hip fracture". It can result from two mechanisms: direct or indirect. Complications include a lack of adhesion and aseptic necrosis of the head of the femur [8]. Eighty percent of trochanteric fractures affect people of older age. Trochanteric fractures can be divided in terms of fracture location: intertrochanteric, transtrochanteric and subtrochanteric. Trochanteric fractures are located in the area between the hip articular capsule and three centimeters below the lesser trochanter. The fracture gap goes through the trabecular bone close to the thigh bone. Good perfusion of this area provides proper healing of fractures, but when a person 
suffers from osteoporosis, prognoses are not optimistic. The limb is shorter and externally rotated after the fracture [8]. In case of transand intertrochanteric fractures, it is suggested to anastomose bones in such way that the limb can be loaded as fast as possible. In the case of fractures that cannot be treated operatively, it is suggested to use direct traction [10].

About $50 \%$ of patients suffering from a neck of femur fracture require long-term care and half of them also require care in specialized medical centers [20]. Fractures are intrinsically painful at the hip area and there is movement limitation. The most essential analysis is based on an X-ray picture of the hip joint. Operational treatment, which enables fast recovery, is suggested. Conservative treatment is related to the risk of high heart rate. Treatment uses operational synthesis. In the case of neck of femur fractures it is suggested to use total hip arthroplasty. Anti-thrombi prophylaxis should be implemented [11]. The strategy of thigh bone fracture treatment is focused on the fastest possible mobilization of a patient [14]. Shortly after an operation, therapists use walking with relief of a limb, active exercises and movement coordination exercises $[15,19]$. Due to the high mortality rate, operational treatment is an optional method and a fracture itself is regarded as life threatening. Patients with neck of femur fractures who cannot be treated operationally should be treated conventionally, with fast mobilization and implementation of chair and bed mode of life [8].

At old age, a thigh bone fracture is a serious problem. $15-20 \%$ of people die during the first 3 months from a trochanteric fracture and $10 \%$ after a neck of femur fracture. About $65 \%$ of persons return to the state before the fracture $[11,12]$.

Fractures of the upper extremity of the humerus apply to its head, anatomical neck, surgical neck, and greater and lesser tubercles. Humerus surgical neck fractures are frequent osteoporotic injuries, especially of persons in their sixties. Generally, they occur due to an indirect mechanism - fall on an upper limb. Pain and edema are present and there are difficulties in movements [8]. At the accident place, the limb should be immobilized, best by bandaging it to the thoracic cavity, immobilizing by a cloth or Kramer's splint [4, 9]. An X-ray picture is a deciding issue in diagnosis. A fractured shoulder is mostly treated conservatively. The limb should be immobilized for a period of 3 to 4 weeks in plaster or stabilizer. Operational treatment is suggested in the case of substantial splinter dislocations and comminuted fractures. Operational treatment implements use of screws and intramedullary nailing. In $6 \%$ of cases, complications occur, especially growth disorders and limited limb movements [10].

\section{Conclusions}

Osteoporotic fractures are becoming a more serious medical problem nowadays. The risk of another fracture increases about 2 to 10 times after the first one. According to the WHO, IOF and EFORT suggestions, general rules of procedures for people suffering from osteoporotic fractures include diagnostics and treatment, informing the patient about osteoporosis and risk of another fracture, implementation of fall preventive procedures, osteoporosis diagnostics, and osteoporosis treatment [25].

Consciousness of serious risks related to osteoporotic fractures should lead to implementation and propagating more active preventive activity for patients of old age. According to the WHO, IOF and EFORT, a doctor admitting a patient with an osteoporotic fracture should not only treat the fracture itself but also implement preventive procedures [25].

\section{Conflict of interest}

The authors declare no conflict of interest.

\section{References}

1. Karl JW, Olson PR, Rosenwasser MP. The epidemiology of upper extremity fractures in the United States, 2009. J Orthop Trauma 2015; 29: e242-4.

2. Cousins S. Exercise, Aging And Health: Overcoming Barriers To An Active Old Age. Taylor \& Francis, 2014.

3. Bakken MS, Engeland A, Engesæter LB, Ranhoff AH, Hunskaar S, Ruths S. Risk of hip fracture among older people using anxiolytic and hypnotic drugs: a nationwide prospective cohort study. Eur J Clin Pharmacol 2014; 70: 873-80.

4. Mazzola P, Bellelli G, Broggini V, et al. Postoperative delirium and pre-fracture disability predict 6-month mortality among the oldest old hip fracture patients. Aging Clin Exp Res 2015; 27: 53-60.

5. Donneys A, Weiss DM, Deshpande SS, et al. Localized deferoxamine injection augments vascularity and improves bony union in pathologic fracture healing after radiotherapy. Bone 2013; 52: 318-25.

6. Zuo D, Zheng L, Sun W, Hua Y, Cai Z. Pathologic fracture does not influence prognosis in stage IIB osteosarcoma: a case-control study. World J Surg Oncol 2013; 11: 148.

7. Sun L, Li Y, Zhang J, Li H, Li B, Ye Z. Prognostic value of pathologic fracture in patients with high grade localized osteosarcoma: a systemic review and meta-analysis of cohort studies. J Orthop Res 2015; 33: 131-9.

8. Murakami K, Yamamoto K, Tsuyuki M, Sugiura T, Tsutsumi S, Kirita T. Theoretical efficacy of preventive measures for pathologic fracture after surgical removal of mandibular lesions based on a three-dimensional finite element analysis. J Oral Maxillof Surg 2014; 72: 833. e1-18.

9. Scolaro JA, Routt ML Jr. Reduction of osteoarticular acetabular dome impaction through an independent iliac cortical window. Injury 2013; 44: 1959-64.

10. Ramirez MA, Ramirez JM, Parks BG, Tsai MA, Murthi AM. Olecranon tip osteoarticular autograft transfer for ir- 
reparable coronoid process fracture: a biomechanical study. Hand 2015; 10: 695-700.

11. Chamouni A, Schreiweis C, Oury F. Bone, brain \& beyond. Rev Endocr Metab Disord 2015; 16: 99-113.

12. Baker A. The three column spine and its significance in the classification of acute thoracolumbar spinal injuries. In: Classic Papers in Orthopaedics. Springer, London 2014; 289-92.

13. Hagen EM. Acute complications of spinal cord injuries. World J Orthop 2015; 6: 17-23.

14. Dubois G, Kheireddine W, Vergari C, et al. Reliable protocol for shear wave elastography of lower limb muscles at rest and during passive stretching. Ultrasound Med Biol 2015; 41: 2284-91.

15. Fadel M, Ahmed MA, Al-Dars AM, Maabed MA, Shawki H. Ilizarov external fixation versus plate osteosynthesis in the management of extra-articular fractures of the distal tibia. Int Orthop 2015; 39: 513-9.

16. Currais A. Ageing and inflammation - a central role for mitochondria in brain health and disease. Ageing Res Rev 2015; 21: 30-42.

17. Fontana L, Kennedy BK, Longo VD, Seals D, Melov S Medical research: treat ageing. Nature News 2014; 511: 405-7.

18. Wloszczak-Szubzda A, Jarosz MJ, Goniewicz M, Goniewicz K. Evaluation of communication and acceptance of the patients by medical personnel. Rocz Państw Zakł Hig 2016; 67: 427-33.

19. Pawłowski, W, Goniewicz K, Goniewicz M, Czerski R. Skull fractures in car accidents: types and causes. J Educ Health Sport 2018; 8: 397-409.

20. Rosengren BE, Karlsson M, Petersson I, Englund M. The $21^{\text {st }}$ - century landscape of adult fractures: cohort study of a complete adult regional population. J Bone Miner Res 2015; 30: 535-42.

21. Roux S, Cabana F, Carrier N, et al. The World Health Organization Fracture Risk Assessment Tool (FRAX) underestimates incident and recurrent fractures in consecutive patients with fragility fractures. J Clin Endocr Metabol 2014; 99: 2400-8.

22. Metcalfe D, Salim A, Olufajo O, et al. Hospital case volume and outcomes for proximal femoral fractures in the USA: an observational study. BMJ Open 2016; 6: e010743.

23. Krztoń-Królewiecka A, Oleszczyk M, Schäfer WLA, Boerma WGW, Windak A. Quality of primary health care in Poland from the perspective of the physicians providing it. BMC Fam Pract 2016; 17: 151.

24. Scott $D$, Seibel $M$, Cumming $R$, et al. Does combined osteopenia/osteoporosis and sarcopenia confer greater risk of falls and fracture than either condition alone in older men? The Concord Health and Ageing in Men Project. J Gerontol A Biol Sci Med Sci 2018 Jul 18. doi: 10.1093/gerona/gly162.

25. Singla R, Gupta Y, Kalra S. Musculoskeletal effects of diabetes mellitus. J Pak Med Assoc 2015; 65: 1024-7. 